\title{
Asymptomatic "giant" pheochromocytoma discovered as adrenal incidentaloma
}

\section{Danijela Radojkovic, Milan Radojkovic, Milica Pesic, Sonja Kostic, Sanja Curkovic, Sasa Radenkovic, Slobodan Antic, Ivan Ilic}

\author{
Medical Faculty, University Nis, Serbia \\ Clinic for endocrinology, diabetes and metabolic disorders, Clinical Center Nis, Serbia
}

Introduction

Adrenal incidentalomas are defined as adrenal lesions incidentally discovered during radiological imaging of the abdomen without prior suspicion of adrenal disease. Approximately $70 \%$ of adrenal incidentalomas are non-functional adenomas. Pheochromocytoma presents only $5-7 \%$ of the remaining functional incidentalomas. Asymptomatic pheochromocytoma-incidentaloma is usually smaller than $1 \mathrm{~cm}$. Large pheochromocytoma, incidentally found, without any clinical signs such as sever hypertension, headache, sweating and tachycardia are very rare.

Patient case report

Young woman ( 32 years old) admitted to

Endocrinology Clinic, after incidentally discovered right adrenal mass, size 6ox7omm, on abdominal sonogram, performed during regular systematic examination. Computed tomography confirmed "giant" tumour, size $70 \times 74 \mathrm{~mm}$, with cystic and necrotic areas and

inhomogeneous contrast captivity.

Patient's medical history of any relevant

illnesses, including hypertension, was negative. She was obese (BMI 32), but without weight gain in the past years. There were no symptoms and signs such as hirsutism, purple

striae, hair loss, or oedema. The blood pressure $(120 / 80 \mathrm{mmHg})$ heart rate $(8 \mathrm{o} / \mathrm{min})$ and respiratory rate (18/min) were normal.

Family history was positive for diabetes mellitus type 2, hypertension and obesity (patient's parents).

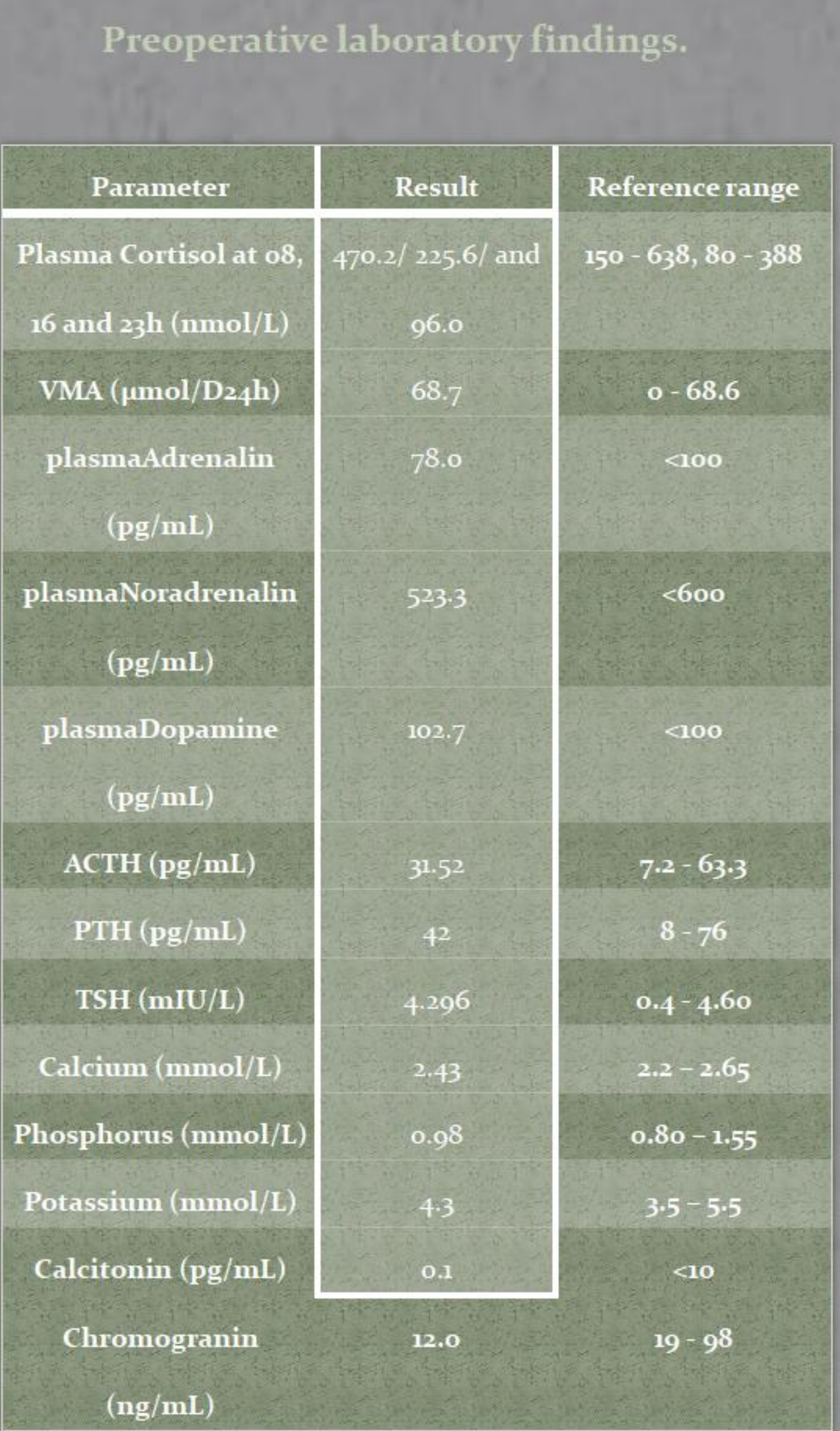

Adrenal gland with tumour

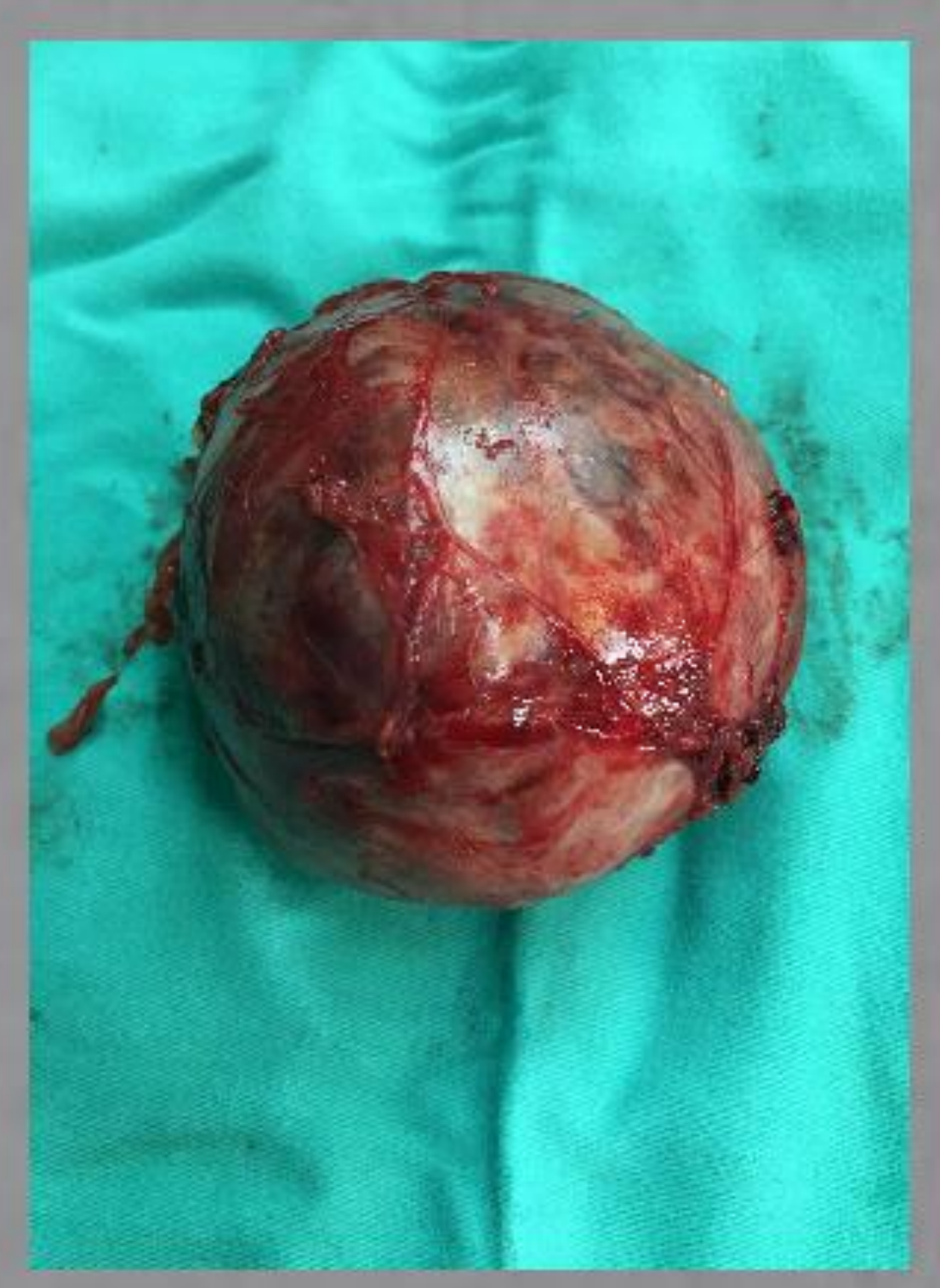

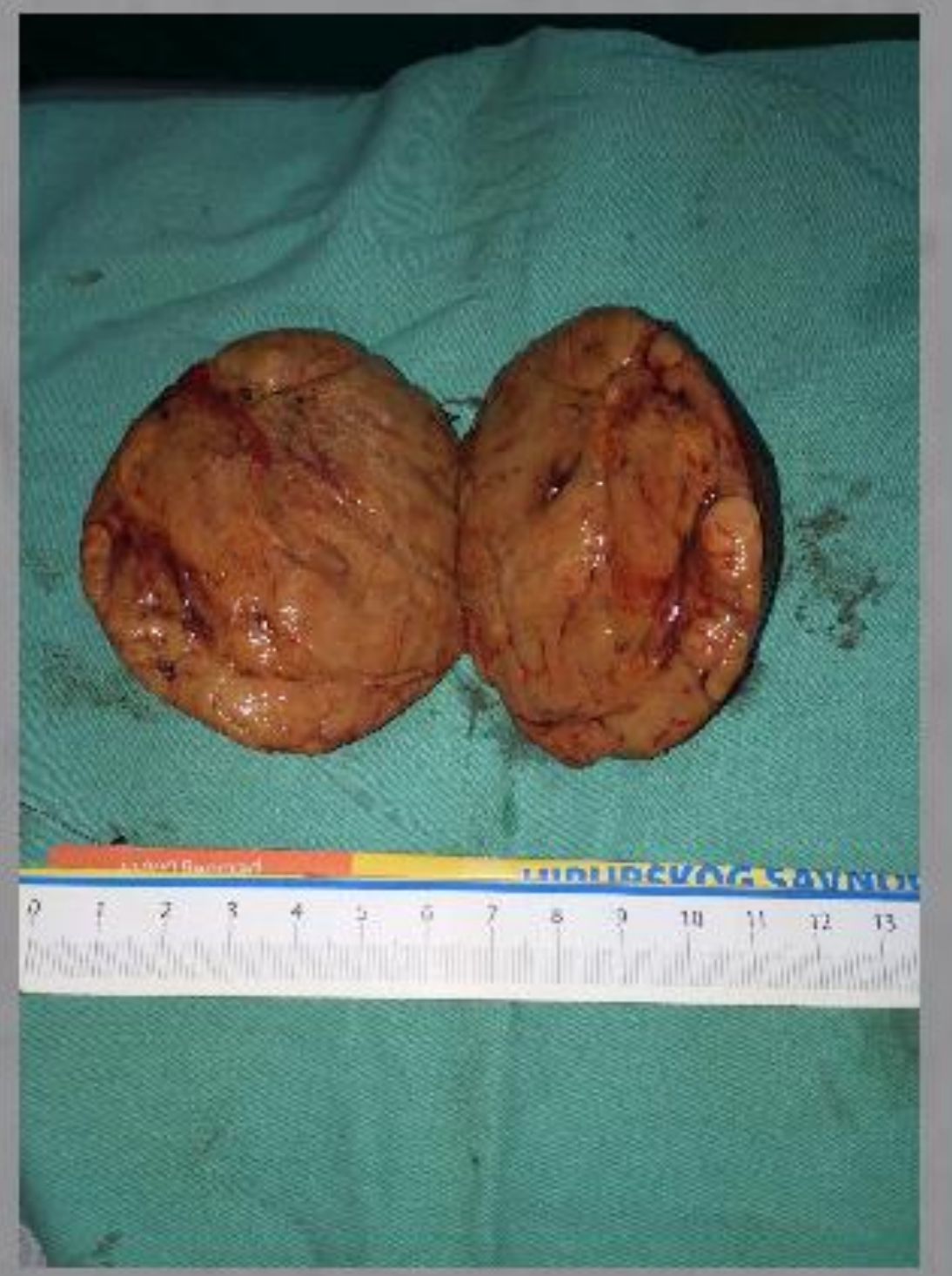

Histopathological examination: benign pheochromocytoma

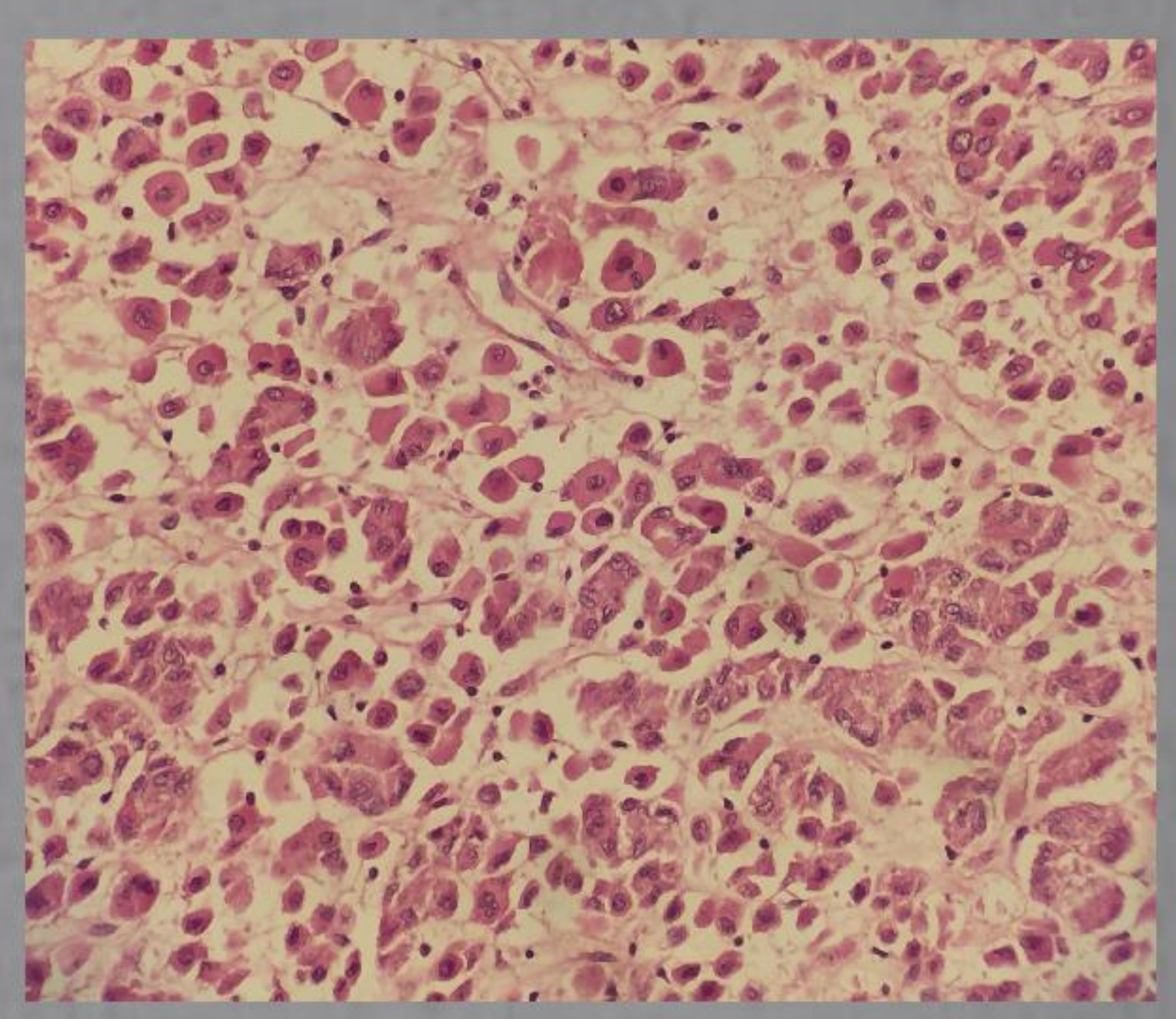

\section{Conclusion}

Due to technological advances the frequency of adrenal incidentaloma diagnosis is constantly increasing. Every incidentally found adrenal mass has to be carefully examined regardless of its clinical presentation in order to prevent fatal oversight of possible secreting nature and/or malignant potential of the lesion and to ensure an adequate curable treatment.

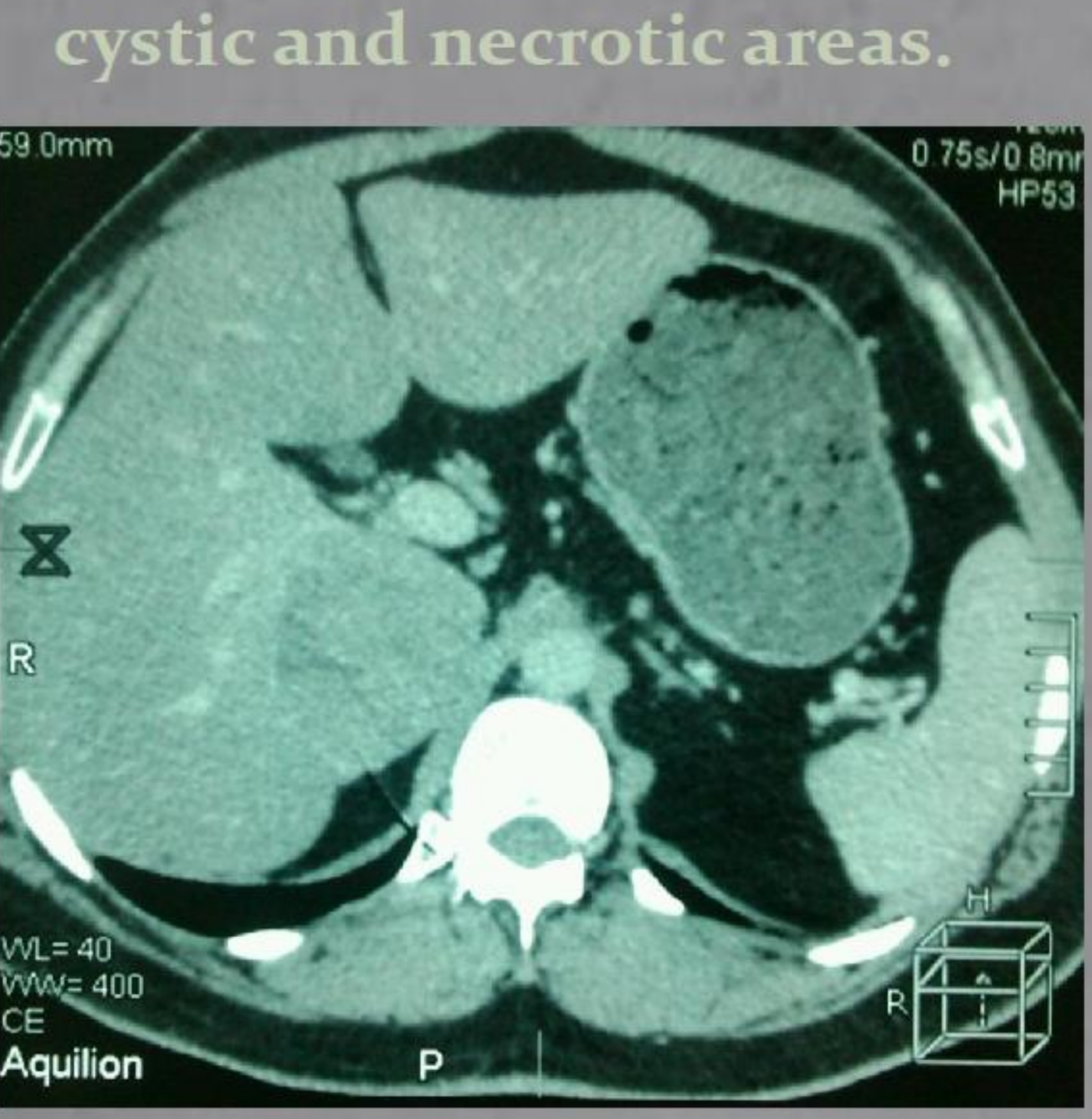

